Review

\title{
Hepatocarcinogenesis by DDT in Rats
}

\author{
Takanori Harada ${ }^{1}$, Ryoichi Ohtsuka ${ }^{1}$, Makio Takeda ${ }^{1}$, Toshinori Yoshida ${ }^{1}$ \\ Akiko Enomoto $^{1}$, Sayuri Kojima ${ }^{1}$, Naruto Tomiyama ${ }^{1}$, \\ Nobuaki Nakashima ${ }^{1}$, and Masakazu Ozaki ${ }^{2}$ \\ ${ }^{1}$ The Institute of Environmental Toxicology, 4321 Uchimoriya-machi, Joso-shi, Ibaraki 303-0043, Japan \\ ${ }^{2}$ Sumika Technoservice Corporation, 1-98 Kasugadenaka, 3-chome Konohana-ku, Osaka 554-8558, Japan
}

\begin{abstract}
DDT is known as a nongenotoxic hepatocarcinogen and has been shown to induce microsomal enzymes and GGT-positive foci and to inhibit gap junctional intercellular communication (GJIC) in the rodent liver. In consideration of these findings, we examined time-related changes in potential factors including hepatic enzyme induction, oxidative stress, cell proliferation, and GJIC in a 4-week and a 2-year feeding studies of p,p'-DDT with F344 rats. Consequently, hepatic microsomal P450 isozymes such as CYP2B1 and CYP3A2 were induced from the beginning of treatment in a dose-dependent manner. Measurement of oxidative stress markers revealed significant increases in lipid peroxide and 8-hydroxydeoxyguanosine at dose levels that induced hepatocellular tumors. Cell proliferation was enhanced within 3 days at any dose level, but returned to normal after 7 days and no significant changes thereafter. GJIC was inhibited throughout the study from the beginning to the end of treatment. Histologically, eosinophilic foci appeared earlier than controls and increased in number and size prior to development of hepatocellular tumors. These results indicate that DDT may induce eosinophilic foci as a result of oxidative DNA damage and leads them to neoplasia in combination with its mitogenic activity and inhibitory effect on GJIC. Oxidative stress could be a key factor in hepatocarcinogenesis by DDT. (J Toxicol Pathol 2006; 19: 155-167)
\end{abstract}

Key words: enzyme induction, oxidative stress, cell proliferation, intercellular communication, eosinophilic foci, DDT

\section{Introduction}

DDT is still used in the tropics and subtropics for the control of malaria and other insect-transmitted diseases, although the use of this compound has been banned or restricted to certain areas in many countries since $1970 \mathrm{~s}$ because of its chemical characteristics such as accumulation and bioconcentration in lipid systems of all animal species which may result in occurrence of potential adverse effects on humans and wild animals. DDT and its metabolites have been extensively studied for their toxicity and carcinogenicity in experimental animals and shown to have a carcinogenic effect on the liver ${ }^{1}$. DDT is classified as a nongenotoxic hepatocarcinogen based on the results of genotoxicity studies ${ }^{1}$ and has been shown to induce microsomal enzymes ${ }^{2-5}$ and GGT-positive foci ${ }^{2}$ and to inhibit gap junctional intercellular communication (GJIC) ${ }^{6-10}$ in the rodent liver. In consideration of these findings, we have conducted further mechanistic studies to

Received: 7 November 2006, Accepted: 10 November 2006

Mailing address: Takanori Harada, The Institute of Environmental Toxicology, 4321 Uchimoriya-machi, Joso-shi, Ibaraki 303-0043, Japan

TEL: 81-297-27-4520 FAX: 81-297-27-4518

E-mail: harada@iet.or.jp examine time-related changes in cell proliferation, GJIC, enzyme induction, oxidative stress and histological alterations including altered hepatocellular foci (AHF) during the carcinogenic process in the liver of F344 rats treated with $p, p^{\prime}-\mathrm{DDT}^{11}$. This paper describes the overview of the time-related changes in these parameters based on our results ${ }^{11}$, and potential factors which might be involved in the hepatocarcinogenesis by DDT are discussed.

\section{Hepatic Microsomal Enzyme Induction}

Our analyses of hepatic microsomal enzymes revealed significant increases in pentoxyresorufin O-dealkylase (PROD) activity and P450 isozyme contents of CYP2B1 and CYP3A2 in the DDT-treated rats (Tables 1-3). The results are generally consistent with previous works ${ }^{2,7,8,12}$. As shown in Tables 1-3, the increases in CYP2B1 and CYP3A2 were dose-dependent, whereas the elevation of PROD activity was most evident in the mid-dose group and not significant in males of the high-dose group after 52, 78, and 104 weeks. This suggests that microsomal enzyme activity is not always consistent with its associated protein content and there seems to be an almost inverse correlation between the increase in PROD at different time points and the 
concurrent incidence of preneoplastic eosinophilic foci and hepatocellular tumors. As for P450 isozyme contents, the increases in CYP2B1 and CYP3A2 tended to be more evident in females than males. A similar result (preferential induction of CYP3A2 in females) also has been reported in Wistar rats treated with the technical grade DDT, a mixture of $p, p^{\prime}$-DDT (85\%), o, $p^{\prime}$-DDT (15\%) and $o, o$ '-DDT (trace amount $)^{5}$. Since CYP3A2 is androgen-dependent and not normally expressed in adult female rats ${ }^{13}$, the induction of CYP3A2 by DDT in female rats suggests that DDT is able to modulate sexual metabolic dimorphism by affecting regulatory sites of hepatic metabolism ${ }^{5}$. The preferential induction of CYP2B1 and CYP3A2 by DDT in female rats indicates an endocrine disrupting potential of DDT because these CYPs are involved in steroid metabolism. With respect to other $\mathrm{P} 450$ isozyme contents, statistically significant increases or decreases in CYP1A2 and CYP4A1 were noted in the DDT-treated groups of both sexes, but those changes were not consistent during the study and their toxicological significance remains obscure.

\section{Oxidative Stress}

Measurements of hepatic oxidative stress markers in our 2-year study disclosed significant increases in lipid peroxide (LPO) and 8-hydroxydeoxyguanosine (8-OHdG) in the mid- and high-dose groups that developed hepatocellular tumors, which were more evident in males than females (Tables 4 and 5). These results indicate that hepatocytes in the DDT-treated livers are exposed to oxidative stress and could have cellular and DNA damages. It is postulated that the metabolic activation with enzyme induction of $\mathrm{P} 450$ by DDT may result in formation of reactive oxygen radicals ${ }^{12,14}$. Since 8-OHdG levels were significantly increased in the DDT-treated groups that developed hepatocellular carcinomas, the increase in $8-\mathrm{OHdG}$ levels (an evidence of oxidative DNA damage) could play an important role in hepatocarcinogenesis by DDT. It has been shown that 8OHdG leads to base mispairing (mutation) on DNA replication ${ }^{15}$.

In our 2-year study, the appearance of eosinophilic altered hepatocellular foci (AHF) in the high-dose group was significantly earlier than that in controls. This result indicates that the occurrence of initiated cells could be accelerated by DDT in the high-dose group, which is considered to be due to hepatocellular DNA damage caused by oxidative stress. It is possible that the accelerated occurrence of initiated cells may result in the early appearance and increased incidence of eosinophilic AHF described later.

\section{Cell Proliferation}

PCNA labeling index (LI) in the liver from rats treated with DDT showed that cell proliferation was enhanced within 3 days of treatment but returned to normal thereafter (Tables 6 and 7). This cell proliferation pattern is consistent with that by nongenotoxic mitogenic hepatocarcinogens ${ }^{16-18}$. It is generally known that the hepatic cell proliferation response to nongenotoxic mitogenic hepatocarcinogens such as phenobarbital typically occurs through an initial burst of enhanced DNA synthesis followed by enhanced mitosis ${ }^{17-19}$. The enhanced cell proliferation, however, ceases after a few days even if treatment is continued. It is considered that an effective feedback mechanism (checkpoint function such as G1 or G2 arrest in cell cycle) may prevent excessive cell multiplication in the normal liver even if the growth stimulatory signals are steadily present due to continuous chemical treatment ${ }^{18}$. It has been postulated that nongenotoxic chemicals with mitogenic activity may provide a selective growth advantage to spontaneously initiated precancerous cells over normal hepatocytes and lead them to neoplasms ${ }^{16,17}$. The increases in the number and size of eosinophilic AHF in our 2-year study could be a reflection of the mitogenic activity of DDT that contributes to the growth of initiated cells.

\section{Gap Junctional Intercellular Communication (GJIC)}

Quantitative analysis of gap junctional intercellular communication (GJIC) in the liver from rats treated with DDT demonstrated a persistent decrease in GJIC protein Cx32 from the beginning to the end of treatment (Tables 8 and 9). GJIC in the liver has been shown to be inhibited by various nongenotoxic tumor-promoting agents including phenobarbital and DDT in vivo and in vitro ${ }^{2,6-8,10,20-22}$. The inhibition of GJIC by tumor promoters may be produced in several ways ${ }^{9}$. Since DDT is highly lipophilic and accumulates in cell membranes, it could interfere directly with the function of GJIC, whereas phenobarbital which is not highly lipophilic may inhibit GJIC in a different way. It is known that GJIC involves the passage of low molecular weight substances between adjacent cells via gap junctions and its function includes the possible regulation of cellular division through cell-to-cell exchange of replication signal molecules ${ }^{7,22,23}$. Therefore, inhibition of GJIC may isolate initiated cells from the growth regulatory signals of neighboring cells and permit the clonal expansion of initiated cells. This suggests an important role for GJIC in the process of tumor formation.

\section{Changes in Liver Weights}

Time-related changes in liver weights in F344 rats treated with DDT are shown in Tables 10-12. Absolute and/ or relative (ratio to body weight) liver weights significantly increased in a dose-dependent manner from the beginning of treatment throughout the study. The liver weight also increased in correlation with duration of exposure but tended to reach a plateau after certain time. It is known that administration of mitogenic agents such as phenobarbital and buthylhydroxytoluene causes an increase in liver weight through mitogenic stimulation of cell proliferation ${ }^{16}$. Upon 


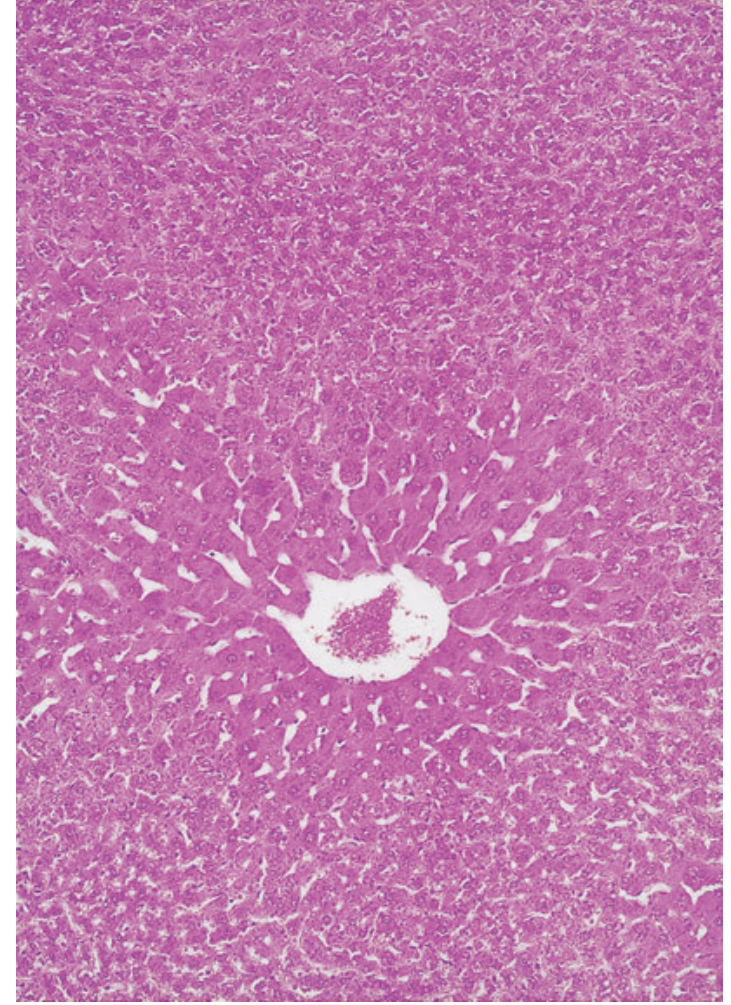

Fig. 1. Centrilobular hepatocellular hypertrophy in a male F344 rat treated with DDT at 500 ppm for 26 weeks. H\&E, $\times 112$.

continued administration, the increased liver weight is maintained, even though the rate of cell turnover returns to normal levels ${ }^{16}$. The increased liver weights in the DDTtreated rats might be due to mitogenic stimulation of cell proliferation to some extent, but the major cause seems to be proliferation of smooth-surfaced endoplasmic reticulum (SER) associated with microsomal enzyme induction since the time of increase in liver weight and enzyme induction was consistent with each other during the study.

\section{Histopathology of the Liver with Morphometry}

In our 2-year study, treatment with DDT induced centrilobular hepatocellular hypertrophy and increased eosinophilic AHF, hepatocellular adenomas and carcinomas (Table 1-3). The severity of hepatocellular hypertrophy (Fig. 1) was dose-dependent and the hypertrophic alteration could be due to SER proliferation associated with microsomal enzyme induction. The eosinophilic AHF noted in the liver treated with DDT typically contained hepatocytes with pale pink or ground glass appearance cytoplasm (Fig. 2) and sometimes had cytoplasmic clear spaces which may represent glycogen deposits. The eosinophilic AHF was positive for GST-P (Fig. 3). The eosinophilic AHF tended to be located in the region close to or within the hypertrophic area. Such a spatial relationship was also reported in rats after continuous administration of low doses of $N$ nitrosomorpholine, which resulted in centrilobular

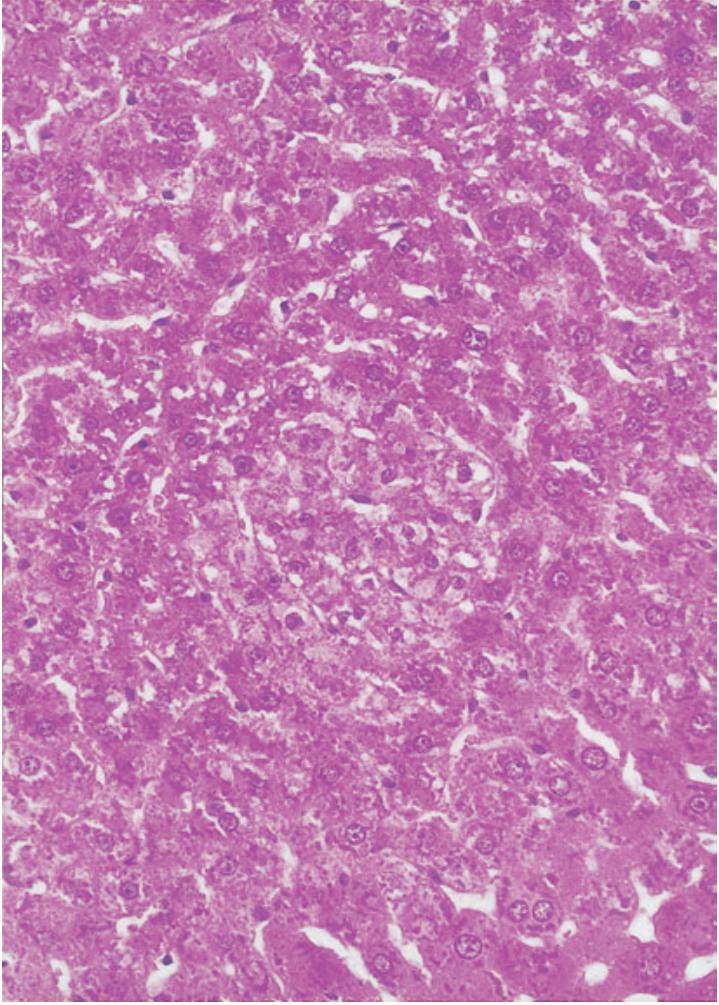

Fig. 2. A small eosinophilic focus in a male F344 rat treated with DDT at 500 ppm for 26 weeks. H\&E, $\times 170$.

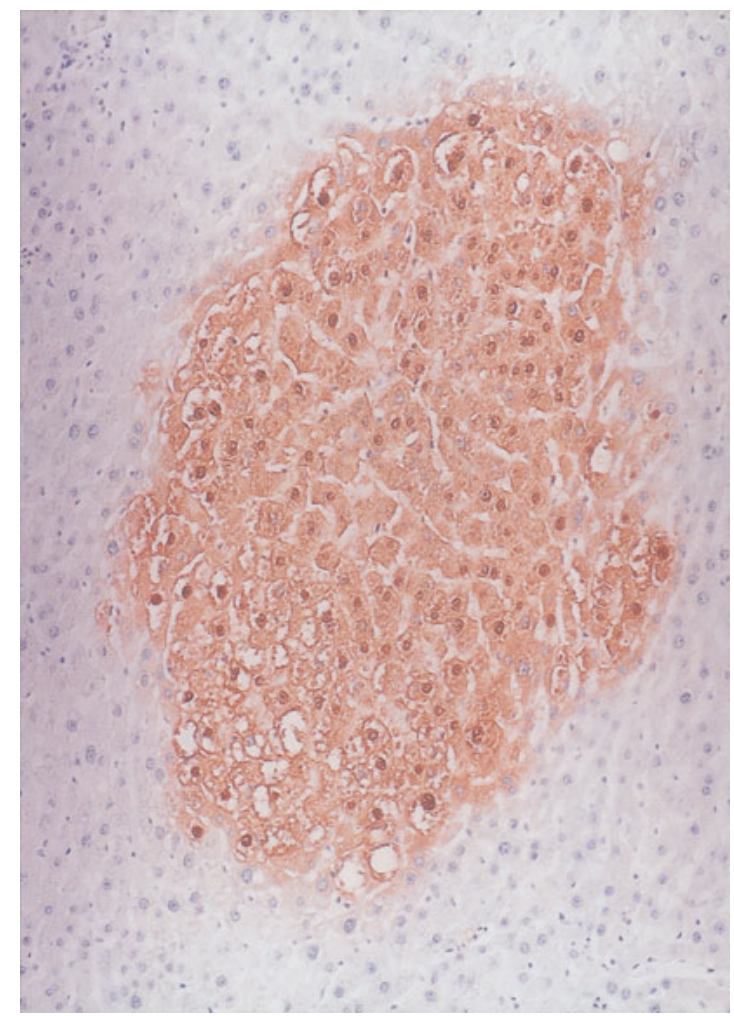

Fig. 3. GST-P positive focus in a male F344 rat treated with DDT at $500 \mathrm{ppm}$ for 52 weeks, which was eosinophilic type of focus in H\&E-stained section. GST-P stain, $\times 112$. 
Table 1. Time-Related Changes in Hepatic Microsomal P450 Isozyme Content in Male F344 Rats from a 4-Week Feeding Study of DDT

\begin{tabular}{|c|c|c|c|c|c|c|}
\hline \multirow{2}{*}{$\begin{array}{l}\text { Dose } \\
\text { (ppm) }\end{array}$} & \multicolumn{6}{|c|}{ CYP2B1 content ( $\mathrm{pmol} / \mathrm{mg}$ protein) on days } \\
\hline & 1 & 2 & 3 & 7 & 14 & 28 \\
\hline 0 & $20.01 \pm 5.41$ & $12.95 \pm 1.23$ & $11.93 \pm 2.93$ & $12.97 \pm 2.77$ & $12.54 \pm 1.73$ & $9.47 \pm 2.41$ \\
\hline 50 & $96.13 \pm 12.70 * *$ & $187.10 \pm 17.52 * *$ & $181.06 \pm 52.39 * *$ & $210.41 \pm 25.13^{* *}$ & $185.19 \pm 36.17 * *$ & $273.32 \pm 48.78 * *$ \\
\hline 160 & $162.45 \pm 19.27 * *$ & $321.44 \pm 33.67 * *$ & $248.15 \pm 27.01 * *$ & $244.15 \pm 33.03^{* *}$ & $329.74 \pm 63.64 * *$ & $252.10 \pm 31.65 * *$ \\
\hline 500 & $166.65 \pm 53.76^{* *}$ & $274.82 \pm 21.13^{* *}$ & $281.66 \pm 45.10^{* *}$ & $280.96 \pm 53.66^{* *}$ & $303.18 \pm 21.92 * *$ & $348.87 \pm 80.59 * *$ \\
\hline
\end{tabular}

Values represent mean \pm S.D. $(n=5)$.

**: Significantly different from control at $\mathrm{p}<0.01$ (Dunnett's multiple comparison test).

Table 2. Time-Related Changes in Hepatic Microsomal Enzyme Activity and P450 Isozyme Contents in Male F344 Rats from a 2-Year Feeding Study of DDT

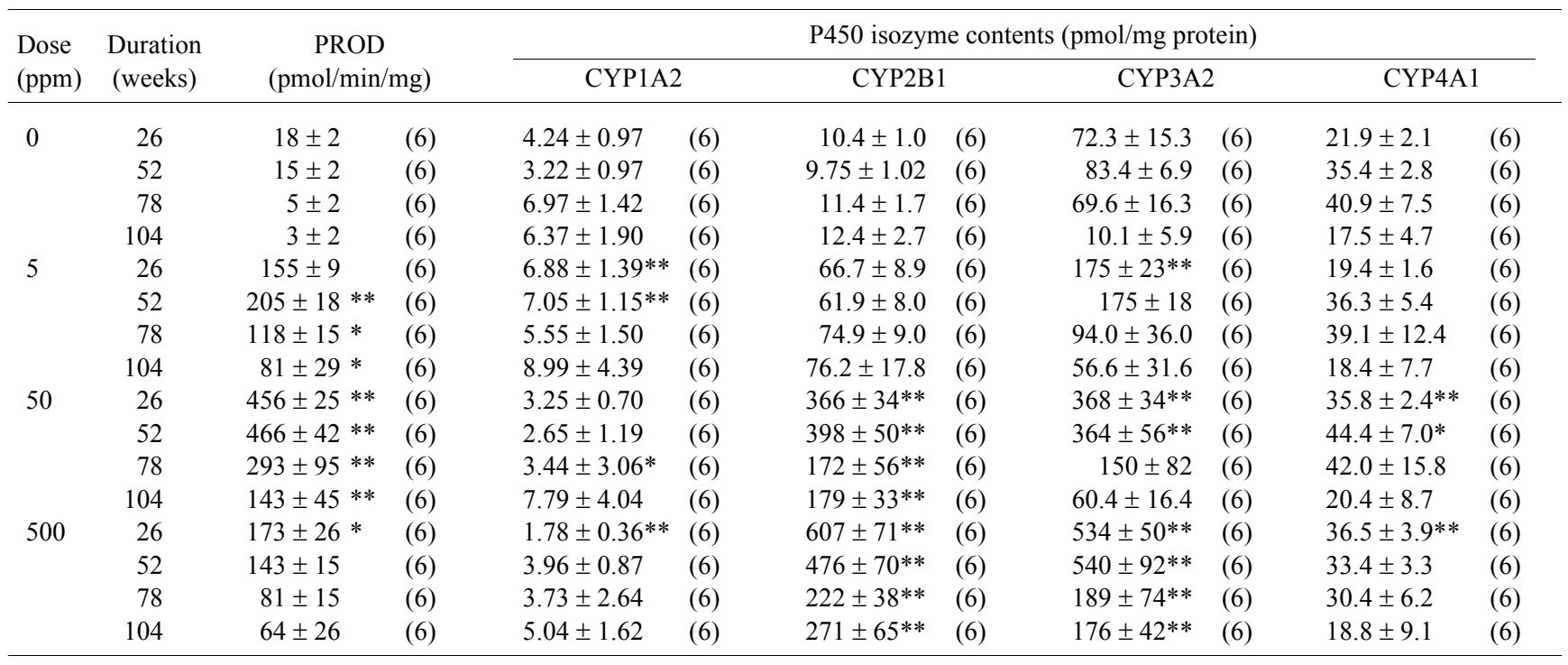

Values represent mean \pm S.D.

(n): Number of animals examined.

*, **: Significantly different from control at $\mathrm{p}<0.05$ and $\mathrm{p}<0.01$, respectively (Dunnett's multiple comparison test).

Table 3. Time-Related Changes in Hepatic Microsomal Enzyme Activity and P450 Isozyme Contents in Female F344 Rats from a 2-Year Feeding Study of DDT

\begin{tabular}{|c|c|c|c|c|c|c|c|c|c|c|c|}
\hline \multirow{3}{*}{$\begin{array}{l}\begin{array}{l}\text { Dose } \\
(\mathrm{ppm})\end{array} \\
0\end{array}$} & \multirow{3}{*}{$\begin{array}{c}\begin{array}{c}\text { Duration } \\
\text { (weeks) }\end{array} \\
26\end{array}$} & \multirow{2}{*}{\multicolumn{2}{|c|}{$\begin{array}{c}\text { PROD } \\
\text { (pmol/min/mg) }\end{array}$}} & \multicolumn{8}{|c|}{$\mathrm{P} 450$ isozyme contents ( $\mathrm{pmol} / \mathrm{mg}$ protein) } \\
\hline & & & & \multicolumn{2}{|c|}{ CYP1A2 } & \multicolumn{2}{|c|}{ CYP2B1 } & \multicolumn{2}{|c|}{ CYP3A2 } & \multicolumn{2}{|c|}{ CYP4A1 } \\
\hline & & $12 \pm 1$ & (6) & $16.8 \pm 6.5$ & (6) & $6.48 \pm 0.94$ & (6) & $10.7 \pm 1.7$ & (6) & $18.8 \pm 2.5$ & (6) \\
\hline & 52 & $8 \pm 2$ & (6) & $21.6 \pm 6.2$ & (6) & $3.81 \pm 1.11$ & (6) & $12.7 \pm 2.1$ & (6) & $14.4 \pm 2.0$ & (6) \\
\hline & 78 & $7 \pm 1$ & (6) & $10.9 \pm 2.9$ & (6) & $4.48 \pm 0.93$ & (6) & $12.1 \pm 2.7$ & (6) & $20.7 \pm 5.1$ & (6) \\
\hline & 104 & $4 \pm 3$ & (6) & $17.9 \pm 8.2$ & (6) & $5.86 \pm 0.77$ & (6) & $7.98 \pm 5.28$ & (6) & $17.9 \pm 5.4$ & (6) \\
\hline \multirow[t]{4}{*}{5} & 26 & $92 \pm 16$ & (6) & $22.6 \pm 4.3$ & (6) & $32.5 \pm 3.6$ & (6) & $16.4 \pm 1.7$ & (6) & $15.8 \pm 2.2$ & (6) \\
\hline & 52 & $95 \pm 26$ & (6) & $29.6 \pm 9.9$ & (6) & $16.9 \pm 4.6$ & (6) & $25.8 \pm 4.4$ & (6) & $20.1 \pm 1.3^{* *}$ & (6) \\
\hline & 78 & $51 \pm 13$ & (6) & $12.0 \pm 3.5$ & (6) & $37.4 \pm 3.7$ & (6) & $29.2 \pm 5.2$ & (6) & $19.2 \pm 4.7$ & (6) \\
\hline & 104 & $50 \pm 18$ & (6) & $17.9 \pm 4.8$ & (6) & $23.8 \pm 6.3$ & (6) & $28.6 \pm 8.4$ & (6) & $22.2 \pm 6.5$ & (6) \\
\hline \multirow[t]{4}{*}{50} & 26 & $402 \pm 74 * *$ & (6) & $23.6 \pm 2.4$ & (6) & $263 \pm 39 * *$ & (6) & $53.5 \pm 9.8^{* *}$ & (6) & $19.5 \pm 2.9$ & (6) \\
\hline & 52 & $393 \pm 44 * *$ & (6) & $31.3 \pm 6.2$ & (6) & $157 \pm 17 * *$ & (6) & $82.3 \pm 8.9^{* *}$ & (6) & $26.1 \pm 3.0^{* *}$ & (6) \\
\hline & 78 & $237 \pm 23^{* *}$ & (6) & $11.5 \pm 4.5$ & (6) & $183 \pm 39 * *$ & (6) & $80.8 \pm 12.4^{* *}$ & (6) & $21.4 \pm 0.5$ & (6) \\
\hline & 104 & $171 \pm 39^{* *}$ & (6) & $14.5 \pm 4.0$ & (6) & $104 \pm 25 * *$ & (6) & $63.1 \pm 11.3 * *$ & (6) & $21.4 \pm 1.7$ & (6) \\
\hline \multirow[t]{4}{*}{500} & 26 & $245 \pm 44^{* *}$ & (6) & $20.6 \pm 4.4$ & (6) & $371 \pm 46^{* *}$ & (6) & $171 \pm 25^{* *}$ & (6) & $14.8 \pm 4.3$ & (6) \\
\hline & 52 & $241 \pm 30 * *$ & (6) & $31.0 \pm 7.2$ & (6) & $350 \pm 29 * *$ & (6) & $155 \pm 21^{* *}$ & (6) & $20.3 \pm 2.9^{* *}$ & (6) \\
\hline & 78 & $106 \pm 10^{* *}$ & (6) & $18.8 \pm 3.5^{* *}$ & (6) & $218 \pm 29 * *$ & (6) & $171 \pm 35^{* *}$ & (6) & $15.7 \pm 3.5$ & (6) \\
\hline & 104 & $90 \pm 25^{*}$ & (5) & $20.6 \pm 1.5$ & (5) & $210 \pm 34 * *$ & (5) & $189 \pm 28 * *$ & (5) & $15.6 \pm 4.8$ & (5) \\
\hline
\end{tabular}

Values represent mean \pm S.D.

(n): Number of animals examined.

*, **: Significantly different from control at $\mathrm{p}<0.05$ and $\mathrm{p}<0.01$, respectively (Dunnett's multiple comparison test). 


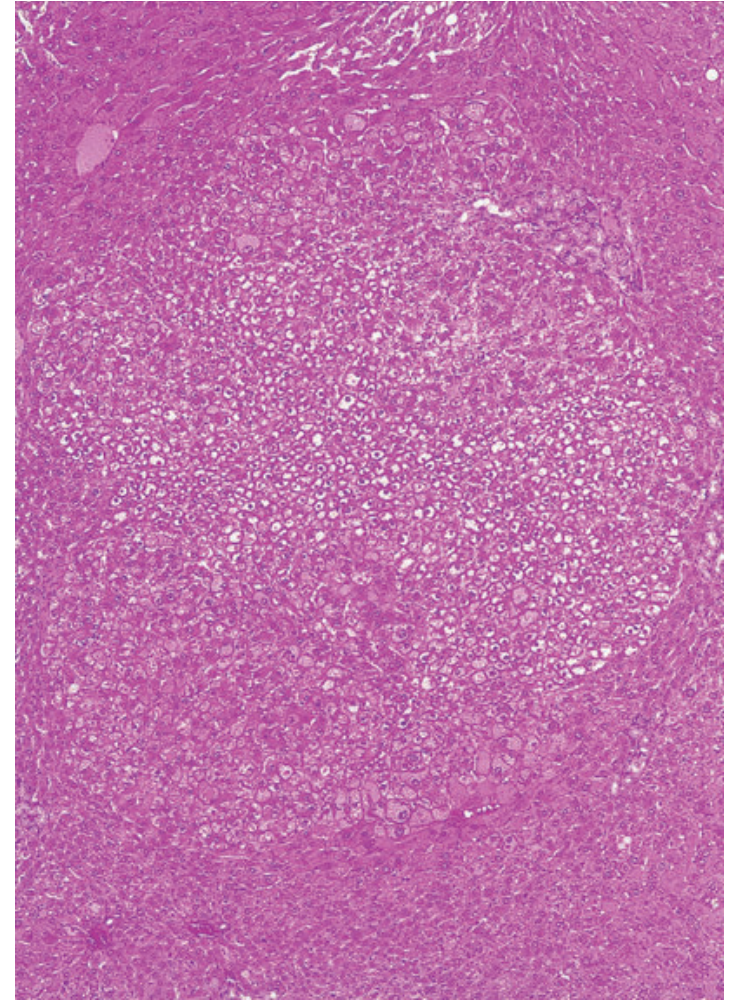

Fig. 4. A large eosinophilic focus in a male F344 rat treated with DDT at 500 ppm for 78 weeks. A small number of basophilic cells are also seen within the lesion. $\mathrm{H} \& \mathrm{E}, \times 54$.

perivenular hepatocellular hypertrophy, being closely related to the later development of preneoplastic hepatic foci including clear/acidophilic foci as well as hepatocellular neoplasms ${ }^{24}$.

Quantitative morphometric analysis revealed that the number and size of eosinophilic AHF were increased in correlation with duration of exposure and dose levels and its appearance was earlier in males than females (Tables 1416). The incidence of eosinophilic AHF in the high-dose group after 104 weeks of treatment reached nearly $100 \%$ for both sexes. Since the first appearance of eosinophilic AHF in the high-dose group (at week 26) was much earlier than that in the controls (at week 78), the eosinophilic preneoplastic lesions could be induced by DDT as a result of DNA damage of hepatocytes exposed to oxidative stress as described before. It is conceivable that DDT may accelerate the occurrence of initiated cells by oxidative stress, leading to the early appearance of eosinophilic AHF and promotes the growth of eosinophilic preneoplastic lesions through its mitogenic activity in combination with the inhibitory effect on GJIC as mentioned before. In other types of AHF, tigroid basophilic AHF decresed in number and size in females in the high dose group. A similar result was also reported in rats treated with phenobarbital ${ }^{21}$.

As to neoplasia, the overall incidences of hepatocellular adenomas and carcinomas in the high-dose group during the study were $55 \%$ and $35 \%$ for males and $40 \%$ and $5 \%$ for

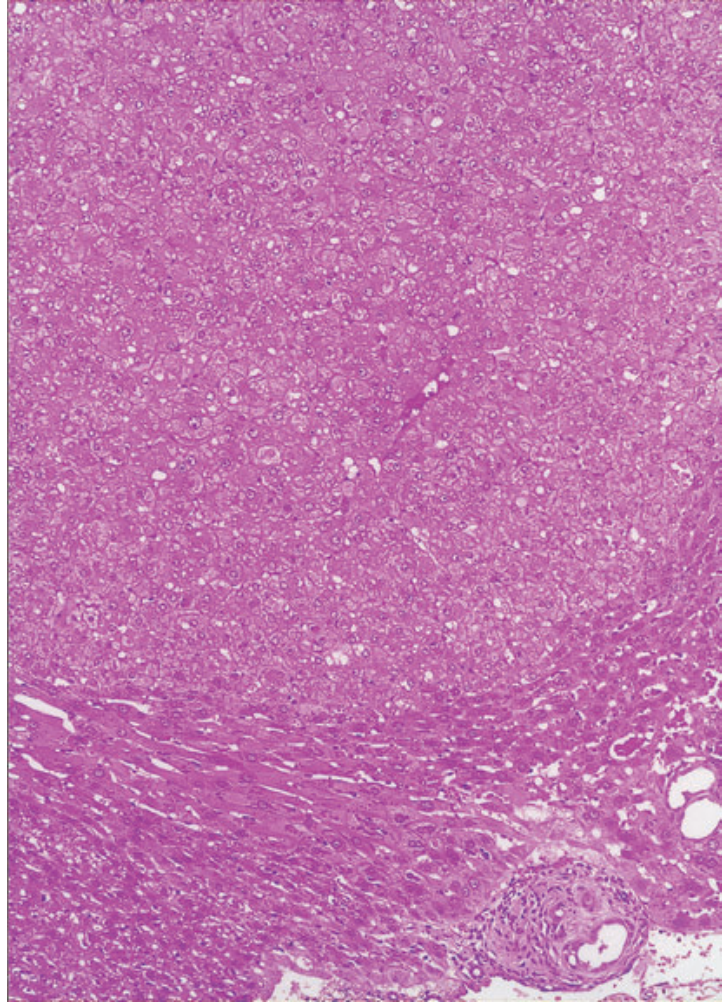

Fig. 5. Hepatocellular adenoma comprising eosinophilic hepatocytes with pale pink or ground glass appearance cytoplasm in a male F344 rat treated with DDT at 500 ppm for 104 weeks. $\mathrm{H} \& \mathrm{E}, \times 85$.

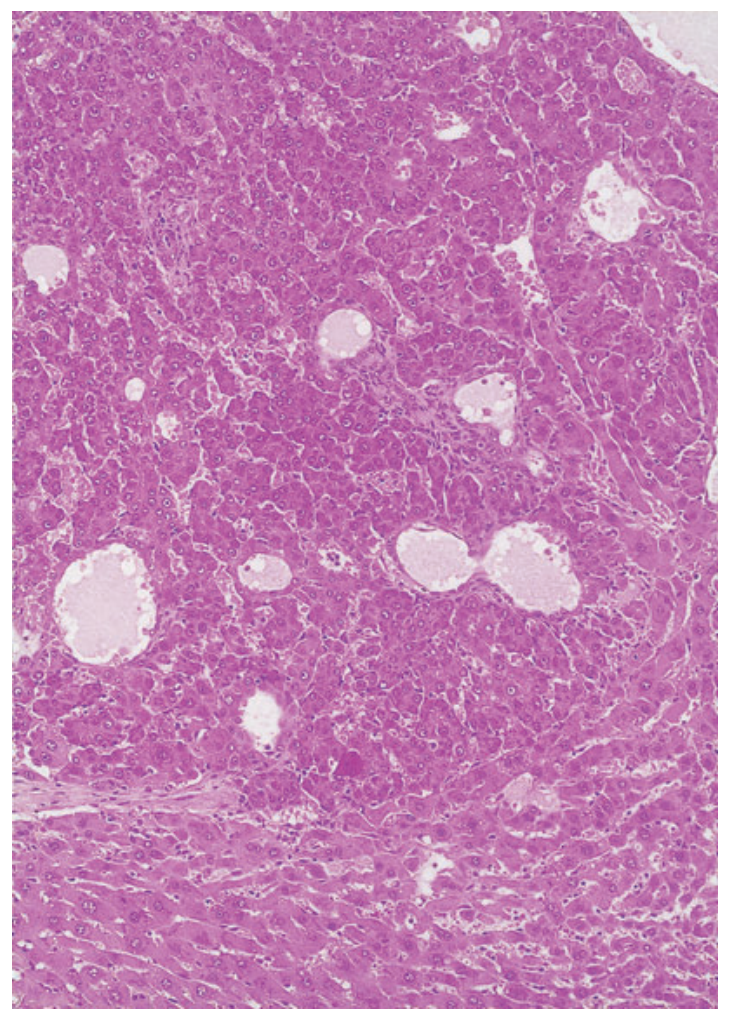

Fig. 6. Hepatocellular carcinoma containing both eosinophilic and basophilic phenotypes of hepatocytes with trabecular pattern in a male F344 rat treated with DDT at $500 \mathrm{ppm}$ for 104 weeks. H\&E, $\times 85$. 
Table 4. Time-Related Changes in Hepatic Lipid Peroxide (LPO) Contents in F344 Rats from a 2-Year Feeding Study of DDT

\begin{tabular}{|c|c|c|c|c|c|c|}
\hline \multirow{2}{*}{$\begin{array}{l}\text { Dose } \\
(\mathrm{ppm})\end{array}$} & \multirow{2}{*}{ Sex } & \multirow{2}{*}{$\begin{array}{c}\text { No. of rats } \\
\text { examined }\end{array}$} & \multicolumn{4}{|c|}{ LPO (nmol/g tissue) at weeks } \\
\hline & & & 26 & 52 & 78 & 104 \\
\hline \multirow[t]{2}{*}{0} & M & 6 & $130 \pm 13$ & $152 \pm 37$ & $157 \pm 37$ & $138 \pm 29$ \\
\hline & $\mathrm{F}$ & 6 & $168 \pm 30$ & $202 \pm 15$ & $150 \pm 21$ & $173 \pm 53$ \\
\hline \multirow[t]{2}{*}{5} & M & 6 & $210 \pm 113$ & $140 \pm 30$ & $217 \pm 71$ & $257 \pm 122$ \\
\hline & $\mathrm{F}$ & 6 & $177 \pm 20$ & $188 \pm 8$ & $157 \pm 43$ & $188 \pm 77$ \\
\hline \multirow[t]{2}{*}{50} & M & 6 & $1005 \pm 324 * *$ & $537 \pm 74 * *$ & $530 \pm 132 * *$ & $523 \pm 332 *$ \\
\hline & $\mathrm{F}$ & 6 & $247 \pm 62^{*}$ & $248 \pm 75$ & $212 \pm 48$ & $307 \pm 78^{*}$ \\
\hline \multirow[t]{2}{*}{500} & M & 6 & $838 \pm 286^{* *}$ & $393 \pm 54 * *$ & $360 \pm 172 *$ & $423 \pm 68 * *$ \\
\hline & $\mathrm{F}$ & 6 & $267 \pm 69 *$ & $178 \pm 24$ & $185 \pm 53$ & $267 \pm 101$ \\
\hline
\end{tabular}

Values represent mean \pm S.D.

*, **: Significantly different from control at $\mathrm{p}<0.05$ and $\mathrm{p}<0.01$, respectively (Dunnett's multiple comparison test).

Table 5. Time-Related Changes in Hepatic 8-OHdG Levels in F344 Rats from a 2-Year Feeding Study of DDT

\begin{tabular}{lcccccc}
\hline \multirow{2}{*}{$\begin{array}{l}\text { Dose } \\
(\mathrm{ppm})\end{array}$} & Sex & $\begin{array}{c}\text { No. of rats } \\
\text { examined }\end{array}$ & \multicolumn{4}{c}{ 8-OHdG (ng/mg DNA) at weeks } \\
\cline { 4 - 6 } 0 & $\mathrm{M}$ & 6 & $4.4 \pm 1.0$ & $3.0 \pm 0.9$ & $2.7 \pm 1.1$ & $3.2 \pm 0.8$ \\
& $\mathrm{~F}$ & 6 & $1.9 \pm 0.5$ & $2.7 \pm 0.5$ & $2.5 \pm 0.5$ & $3.0 \pm 0.8$ \\
5 & $\mathrm{M}$ & 6 & $3.7 \pm 1.3$ & $3.8 \pm 0.8$ & $2.2 \pm 0.7$ & $3.5 \pm 0.7$ \\
& $\mathrm{~F}$ & 6 & $2.0 \pm 0.2$ & $4.0 \pm 1.9$ & $2.7 \pm 0.3$ & $2.9 \pm 0.6$ \\
50 & $\mathrm{M}$ & 6 & $5.9 \pm 1.6$ & $6.1 \pm 2.8$ & $2.9 \pm 1.2$ & $5.0 \pm 1.0$ \\
& $\mathrm{~F}$ & 6 & $3.0 \pm 1.6$ & $5.2 \pm 1.4$ & $3.1 \pm 0.4$ & $2.7 \pm 0.5$ \\
500 & $\mathrm{M}$ & 6 & $15.4 \pm 4.5^{* *}$ & $13.8 \pm 4.3^{* *}$ & $7.5 \pm 2.2^{* *}$ & $15.9 \pm 9.9^{* *}$ \\
& $\mathrm{~F}$ & 6 & $2.6 \pm 1.1$ & $14.1 \pm 5.2^{* *}$ & $6.2 \pm 2.9^{* *}$ & $5.8 \pm 0.9^{* *}$ \\
\hline
\end{tabular}

Values represent mean \pm S.D.

**: Significantly different from control at $\mathrm{p}<0.01$ (Dunnett's multiple comparison test).

Table 6. Time-Related Changes in PCNA LI (\%) in the Liver of Male F344 Rats from a 4-Week Feeding Study of DDT

\begin{tabular}{lcccccc}
\hline $\begin{array}{l}\text { Dose } \\
(\mathrm{ppm})\end{array}$ & \multicolumn{5}{c}{ PCNA LI (mean $\pm \mathrm{SD}$ ) on days } \\
\cline { 2 - 6 } & 1 & 2 & 3 & 7 & 14 & 28 \\
\hline 0 & $2.00 \pm 0.80$ & $1.58 \pm 0.68$ & $3.19 \pm 1.44$ & $4.46 \pm 1.41$ & $1.75 \pm 0.77$ & $0.96 \pm 0.50$ \\
& $(5)$ & $(5)$ & $(5)$ & $(5)$ & $(5)$ & $(5)$ \\
50 & $2.06 \pm 0.46$ & $3.22 \pm 0.51^{*}$ & $4.84 \pm 1.60$ & $5.37 \pm 0.36$ & $0.96 \pm 0.31$ & $0.58 \pm 0.18$ \\
& $(5)$ & $(5)$ & $(5)$ & $(5)$ & $(5)$ & $(5)$ \\
160 & $2.81 \pm 0.67$ & $5.91 \pm 1.49^{* *}$ & $6.85 \pm 1.71^{* *}$ & $4.13 \pm 0.72$ & $0.62 \pm 0.54$ & $0.60 \pm 0.34$ \\
& $(5)$ & $(5)$ & $(5)$ & $(5)$ & $(5)$ & $(5)$ \\
500 & $4.62 \pm 1.01^{* *}$ & $5.33 \pm 0.59^{* *}$ & $6.31 \pm 1.57^{*}$ & $4.52 \pm 1.27$ & $1.39 \pm 1.19$ & $0.94 \pm 0.48$ \\
& $(5)$ & $(5)$ & $(5)$ & $(5)$ & $(5)$ & $(5)$ \\
\hline
\end{tabular}

(n): Number of animals examined.

*,**: Significantly different from control at $\mathrm{p}<0.05$ and $\mathrm{p}<0.01$, respectively (Dunnett's multiple comparison test).

females, respectively (Table 17). Morphologically, the hepatocellular adenomas contained typically eosinophilic hepatocytes (Fig. 5) which were similar to those of eosinophilc AHF described above and often had basophilic phenotypes in small numbers. Basophilic cell type of adenomas was also noted but only in a few animals. On the other hand, the hepatocellular carcinomas contained hepatocytes with eosinophilic or basophilic cytoplasm and typically had admixture of both phenotypes (Fig. 6). The population of basophilic cells in the carcinomas was much higher than that in adenomas or large eosinophilic AHF. Since the hepatocytes within the majority of adenomas were morphologically similar to those of eosinophilic AHF and there was no increase in other types of AHF, it was suggested that the eosinophilic AHF could develop into neoplasms without passing an intermediate stage ${ }^{25}$. However, large eosinophic AHF and adenomas sometimes contained small number of basophilic cells within the 
Table 7. Time-Related Changes in PCNA LI (\%) in the Liver of F344 Rats from a 2-Year

Table 7. Time-Related Changes in
Feeding Study of DDT

\begin{tabular}{lccccc}
\hline \multirow{2}{*}{$\begin{array}{l}\text { Dose } \\
(\mathrm{ppm})\end{array}$} & Sex & \multicolumn{4}{c}{ PCNA LI (mean \pm SD) at weeks } \\
\cline { 3 - 6 } & & 26 & 52 & 78 & 104 \\
\hline 0 & $\mathrm{M}$ & $0.70 \pm 0.34$ & $0.43 \pm 0.11$ & $0.82 \pm 0.63$ & $0.80 \pm 1.03$ \\
& & $(6)$ & $(6)$ & $(6)$ & $(5)$ \\
& $\mathrm{F}$ & $0.25 \pm 0.22$ & $0.17 \pm 0.11$ & $0.93 \pm 0.52$ & $0.45 \pm 0.32$ \\
& & $(6)$ & $(6)$ & $(6)$ & $(5)$ \\
5 & $\mathrm{M}$ & $0.67 \pm 0.38$ & $0.40 \pm 0.22$ & $0.69 \pm 0.25$ & $0.43 \pm 0.19$ \\
& & $(6)$ & $(6)$ & $(6)$ & $(5)$ \\
& $\mathrm{F}$ & $0.13 \pm 0.05$ & $0.14 \pm 0.08$ & $1.13 \pm 1.17$ & $0.28 \pm 0.07$ \\
& & $(6)$ & $(6)$ & $(6)$ & $(6)$ \\
50 & $\mathrm{M}$ & $0.77 \pm 0.19$ & $0.28 \pm 0.13$ & $0.75 \pm 0.31$ & $0.42 \pm 0.21$ \\
& & $(6)$ & $(6)$ & $(6)$ & $(4)$ \\
& $\mathrm{F}$ & $0.87 \pm 0.69$ & $0.36 \pm 0.16$ & $1.10 \pm 0.59$ & $0.46 \pm 0.25$ \\
& & $(6)$ & $(6)$ & $(6)$ & $(6)$ \\
500 & $\mathrm{M}$ & $0.32 \pm 0.22$ & $0.29 \pm 0.16$ & $0.78 \pm 0.24$ & $0.73 \pm 0.48$ \\
& & $(6)$ & $(6)$ & $(6)$ & $(6)$ \\
& $\mathrm{F}$ & $0.45 \pm 0.18$ & $0.34 \pm 0.31$ & $0.62 \pm 0.20$ & $0.55 \pm 0.06$ \\
& & $(6)$ & $(6)$ & $(6)$ & $(4)$ \\
\hline
\end{tabular}

(n): Number of animals examined.

Table 8. Time-Related Changes in the Number of GJIC Protein Cx32 Spots in the Liver of Male F344 Rats from a 4-Week Feeding Study of DDT

\begin{tabular}{|c|c|c|c|c|c|c|}
\hline \multirow{2}{*}{$\begin{array}{l}\text { Dose } \\
(\mathrm{ppm})\end{array}$} & \multicolumn{6}{|c|}{ No. of Cx32 spots (mean \pm SD) on days } \\
\hline & 1 & 2 & 3 & 7 & 14 & 28 \\
\hline 0 & $\begin{array}{c}8.29 \pm 0.36 \\
(5)\end{array}$ & $\begin{array}{c}7.81 \pm 0.75 \\
(5)\end{array}$ & $\begin{array}{c}7.86 \pm 0.81 \\
(5)\end{array}$ & $\begin{array}{c}7.24 \pm 0.38 \\
(5)\end{array}$ & $\begin{array}{c}7.64 \pm 0.52 \\
(5)\end{array}$ & $\begin{array}{c}8.11 \pm 0.22 \\
(5)\end{array}$ \\
\hline 50 & $\begin{array}{c}5.59 \pm 0.77 * * \\
(5)\end{array}$ & $4.69 \pm 0.33 * *$ & $\begin{array}{c}5.41 \pm 0.40 * * \\
(5)\end{array}$ & $\begin{array}{c}3.14 \pm 0.33 * * \\
(5)\end{array}$ & $\begin{array}{c}2.99 \pm 0.53 * * \\
(5)\end{array}$ & $3.33 \pm 0.74 * *$ \\
\hline 160 & $\begin{array}{c}4.90 \pm 0.63 * * \\
(5)\end{array}$ & $\begin{array}{c}4.37 \pm 0.64 * * \\
(5)\end{array}$ & $\begin{array}{c}3.94 \pm 0.64 * * \\
(5)\end{array}$ & $\begin{array}{c}3.06 \pm 0.46 * * \\
(5)\end{array}$ & $\begin{array}{c}2.71 \pm 0.68 * * \\
(5)\end{array}$ & $2.77 \pm 0.43 * *$ \\
\hline 500 & $\begin{array}{c}5.35 \pm 1.03 * * \\
(5)\end{array}$ & $\begin{array}{c}3.98 \pm 0.41 * * \\
(5)\end{array}$ & $\begin{array}{c}3.12 \pm 0.33 * * \\
(5)\end{array}$ & $\begin{array}{c}2.62 \pm 0.70 * * \\
(5)\end{array}$ & $\begin{array}{c}1.75 \pm 0.51 * * \\
(5)\end{array}$ & $\begin{array}{c}1.68 \pm 0.79 * * \\
(5)\end{array}$ \\
\hline
\end{tabular}

(n): Number of animals examined.

**: Significantly different from control at $\mathrm{p}<0.01$ (Dunnett's multiple comparison test).

Table 9. Time-Related Changes in the Number of GJIC Protein Cx32 Spots in the Liver of F344 Rats from a 2-Year Feeding Study of DDT

\begin{tabular}{|c|c|c|c|c|c|}
\hline \multirow{2}{*}{$\begin{array}{l}\text { Dose } \\
(\mathrm{ppm})\end{array}$} & \multirow{2}{*}{ Sex } & \multicolumn{4}{|c|}{ No. of Cx32 spots (mean \pm SD) at weeks } \\
\hline & & 26 & 52 & 78 & 104 \\
\hline \multirow[t]{2}{*}{0} & M & $\begin{array}{c}7.07 \pm 0.94 \\
(6)\end{array}$ & $\begin{array}{c}6.49 \pm 1.46 \\
(6)\end{array}$ & $\begin{array}{c}3.05 \pm 0.19 \\
(6)\end{array}$ & $\begin{array}{c}3.11 \pm 1.15 \\
(5)\end{array}$ \\
\hline & $\mathrm{F}$ & $\begin{array}{c}6.08 \pm 0.97 \\
(6)\end{array}$ & $\begin{array}{c}6.48 \pm 0.66 \\
(6)\end{array}$ & $\begin{array}{c}3.26 \pm 0.74 \\
(6)\end{array}$ & $\begin{array}{c}3.73 \pm 0.23 \\
(5)\end{array}$ \\
\hline \multirow[t]{2}{*}{5} & M & $\begin{array}{c}6.87 \pm 0.54 \\
(6)\end{array}$ & $\begin{array}{c}6.95 \pm 0.88 \\
(6)\end{array}$ & $\begin{array}{c}2.51 \pm 0.50 * \\
\quad(6)\end{array}$ & $\begin{array}{c}2.13 \pm 0.37 \\
(5)\end{array}$ \\
\hline & $\mathrm{F}$ & $\begin{array}{c}5.67 \pm 0.93 \\
(6)\end{array}$ & $\begin{array}{c}5.51 \pm 1.62 \\
(6)\end{array}$ & $\begin{array}{c}2.90 \pm 0.41 \\
(6)\end{array}$ & $\begin{array}{c}3.59 \pm 0.42 \\
(6)\end{array}$ \\
\hline \multirow[t]{2}{*}{50} & M & $\begin{array}{c}5.53 \pm 0.83 * \\
(6)\end{array}$ & $\begin{array}{c}6.28 \pm 1.88 \\
(6)\end{array}$ & $\begin{array}{c}2.47 \pm 0.44 * \\
(6)\end{array}$ & $\begin{array}{c}2.13 \pm 0.35 \\
(4)\end{array}$ \\
\hline & $\mathrm{F}$ & $\begin{array}{c}4.80 \pm 0.86 \\
(6)\end{array}$ & $\begin{array}{c}4.20 \pm 0.56^{* *} \\
(6)\end{array}$ & $\begin{array}{c}1.76 \pm 0.69 * \\
(6)\end{array}$ & $\begin{array}{c}2.65 \pm 0.33 * * \\
(6)\end{array}$ \\
\hline \multirow[t]{2}{*}{500} & M & $\begin{array}{c}5.39 \pm 1.30 * \\
(6)\end{array}$ & $\begin{array}{c}4.64 \pm 0.91 \\
(6)\end{array}$ & $\begin{array}{c}1.14 \pm 0.22 * * \\
(6)\end{array}$ & $\begin{array}{c}1.52 \pm 0.28 * \\
(6)\end{array}$ \\
\hline & $\mathrm{F}$ & $\begin{array}{c}4.20 \pm 1.41 * \\
(6)\end{array}$ & $\begin{array}{c}3.28 \pm 0.72 * * \\
(6)\end{array}$ & $\begin{array}{c}1.03 \pm 0.16 * * \\
(6)\end{array}$ & $\begin{array}{c}1.75 \pm 0.47 * * \\
(4)\end{array}$ \\
\hline
\end{tabular}

(n): Number of animals examined.

*, **: Significantly different from control at $\mathrm{p}<0.05$ and $\mathrm{p}<0.01$, respectively (Dunnett's multiple comparison test). 
Table 10. Time-Related Changes in Liver Weights of Male F344 Rats from a 4-Week Feeding Study of DDT

\begin{tabular}{|c|c|c|c|c|c|c|c|}
\hline \multirow{2}{*}{$\begin{array}{l}\text { Dose } \\
(\mathrm{ppm})\end{array}$} & \multirow{2}{*}{ Liver weights } & \multicolumn{6}{|c|}{ Days on study } \\
\hline & & 1 & 2 & 3 & 7 & 14 & 28 \\
\hline \multirow[t]{2}{*}{50} & Absolute & $109(5)$ & $107(5)$ & $106(5)$ & $112(5)^{*}$ & $126(5)^{*}$ & $112(5) * *$ \\
\hline & Relative & $108(5)^{* *}$ & $107(5)$ & $107(5)^{*}$ & $111(5)^{*}$ & $121(5)^{* *}$ & $110(5)^{* *}$ \\
\hline \multirow[t]{2}{*}{160} & Absolute & $105(5)$ & $114(5)$ & $121(5)^{* *}$ & $127(5)^{* *}$ & $142(5)^{* *}$ & $124(5) * *$ \\
\hline & Relative & $107(5)^{*}$ & $113(5)^{* *}$ & $119(5)^{* *}$ & $123(5)^{* *}$ & $136(5)^{* *}$ & $122(5)^{* *}$ \\
\hline \multirow[t]{2}{*}{500} & Absolute & $110(5)$ & $125(5)^{* *}$ & $127(5)^{* *}$ & $153(5)^{* *}$ & $148(5)^{* *}$ & $142(5) * *$ \\
\hline & Relative & $111(5)^{* *}$ & $125(5)^{* *}$ & $129(5)^{* *}$ & $147(5)^{* *}$ & $148(5)^{* *}$ & $145(5)^{* *}$ \\
\hline
\end{tabular}

Values represent percentages (\%) to control values.

(n): Number of animals examined.

Relative: Ratio to body weight (\%)

*,**: Significantly different from control at $\mathrm{p}<0.05$ and $\mathrm{p}<0.01$, respectively (Dunnett's multiple comparison test).

Table 11. Time-Related Changes in Liver Weights of Male F344 Rats from a 2-Year Feeding Study of DDT

\begin{tabular}{|c|c|c|c|c|c|}
\hline \multirow{2}{*}{$\begin{array}{l}\text { Dose } \\
(\mathrm{ppm})\end{array}$} & \multirow{2}{*}{ Liver weights } & \multicolumn{3}{|c|}{ Weeks on study } & \multirow[b]{2}{*}{104} \\
\hline & & 26 & 52 & 78 & \\
\hline \multirow[t]{2}{*}{5} & Absolute & $106(6)$ & $108(6)$ & $108(8)$ & $110(10)$ \\
\hline & Relative & $102(6)$ & $108(6)$ & $105(8)$ & $105(10)$ \\
\hline \multirow[t]{2}{*}{50} & Absolute & $114(6)^{* *}$ & $117(6)^{*}$ & $126(8)^{* *}$ & $125(10)^{* *}$ \\
\hline & Relative & $112(6)^{*}$ & $118(6)^{*}$ & $125(8)^{* *}$ & $123(10)^{*}$ \\
\hline \multirow[t]{2}{*}{500} & Absolute & $166(6)^{* *}$ & $154(6)^{* *}$ & $153(7)^{* *}$ & $149(10)^{* *}$ \\
\hline & Relative & $170(6)^{* *}$ & $168(6)^{* *}$ & $166(7)^{* *}$ & $171(10)^{* *}$ \\
\hline
\end{tabular}

Values represent percentages (\%) to control values.

(n): Number of animals examined.

Relative: Ratio to body weight (\%)

*,**: Significantly different from control at $\mathrm{p}<0.05$ and $\mathrm{p}<0.01$, respectively (Dunnett's multiple comparison test).

Table 12. Time-Related Changes in Liver Weights of Female F344 Rats from a 2-Year Feeding Study of DDT

\begin{tabular}{|c|c|c|c|c|c|}
\hline \multirow{2}{*}{$\begin{array}{l}\text { Dose } \\
(\mathrm{ppm})\end{array}$} & \multirow{2}{*}{ Liver weights } & \multicolumn{4}{|c|}{ Weeks on study } \\
\hline & & 26 & 52 & 78 & 104 \\
\hline \multirow[t]{2}{*}{5} & Absolute & $109(6)$ & $106(6)$ & $104(8)$ & $102(10)$ \\
\hline & Relative & $106(6)$ & $103(6)$ & $103(8)$ & $101(10)$ \\
\hline \multirow[t]{2}{*}{50} & Absolute & $111(6)^{*}$ & $123(6)$ & $110(7)$ & $111(10)$ \\
\hline & Relative & $114(6)^{*}$ & $118(6)^{* *}$ & $109(7)$ & $110(10)$ \\
\hline \multirow[t]{2}{*}{500} & Absolute & $146(6)^{* *}$ & $151(6)^{* *}$ & $143(8)^{* *}$ & $144(10)^{* *}$ \\
\hline & Relative & $163(6)^{* *}$ & $170(6)^{* *}$ & $165(8)^{* *}$ & $190(10)^{* *}$ \\
\hline
\end{tabular}

Values represent percentages (\%) to control values.

(n): Number of animals examined.

Relative: Ratio to body weight (\%)

$*$, **: Significantly different from control at $\mathrm{p}<0.05$ and $\mathrm{p}<0.01$, respectively (Dunnett's multiple comparison test).

lesions. The presence of basophilic cells might be an indication of malignant transformation of hepatocytes within the lesions since the population of basophilic phenotypes is largest in the hepatocellular carcinomas ${ }^{25}$. A similar type of nongenotoxic hepatocarcinogen, phenobarbital (PB), also has been shown to induce eosinophilic altered foci and hepatocellular tumors containing eosinophilic cells (GGT positive $)^{21}$. However, it should be recognized that the occurrence of eosinophilic AHF is not limited to PB and DDT because many other chemicals including genotoxic agents also induced such eosinophilic preneoplastic lesions ${ }^{25,26}$.

With respect to sex difference in tumor development, it is generally believed that males have a higher incidence of hepatic tumors than females in rodents as well as in humans ${ }^{27}$. Factors contributing to this sex difference have not been clearly demonstrated, but it may be due to the difference of hormonal pattern which is of primary importance for determining the metabolic activation of carcinogens and also due to the sex chromosome in its role as 
Table 13. Incidences of Hepatocellular Hypertrophy and Altered Hepatocellular Foci (AHF) in F344 Rats from a 2-Year Feeding Study of DDT

\begin{tabular}{|c|c|c|c|c|c|c|c|c|c|}
\hline \multirow{2}{*}{$\begin{array}{l}\text { Dose } \\
(\mathrm{ppm})\end{array}$} & \multirow{2}{*}{$\begin{array}{r}\text { Duration } \\
\text { (weeks) }\end{array}$} & \multicolumn{2}{|c|}{ Hypertrophy } & \multicolumn{2}{|c|}{ Eosinophilic AHF } & \multicolumn{2}{|c|}{ Tigroid basophilic AHF } & \multicolumn{2}{|c|}{ Clear cell AHF } \\
\hline & & Male & Female & Male & Female & Male & Female & Male & Female \\
\hline \multirow[t]{5}{*}{0} & 26 & $0 / 6$ & $0 / 6$ & $0 / 6$ & $0 / 6$ & $2 / 6$ & $2 / 6$ & $0 / 6$ & $0 / 6$ \\
\hline & 52 & $0 / 6$ & $0 / 6$ & $0 / 6$ & $0 / 6$ & $3 / 6$ & $4 / 6$ & $5 / 6$ & $0 / 6$ \\
\hline & 78 & $0 / 8$ & $0 / 8$ & $1 / 8$ & $0 / 8$ & $8 / 8$ & $8 / 8$ & $5 / 8$ & $3 / 8$ \\
\hline & 104 & $0 / 35$ & $0 / 33$ & $23 / 35$ & $6 / 33$ & $34 / 35$ & $30 / 33$ & $30 / 35$ & $8 / 33$ \\
\hline & Total $^{\mathrm{a}}$ & $0 / 40$ & $0 / 40$ & $24 / 40$ & $8 / 40$ & $37 / 40$ & $37 / 40$ & $30 / 40$ & $9 / 40$ \\
\hline \multirow[t]{5}{*}{5} & 26 & $2 / 6$ & $0 / 6$ & $0 / 6$ & $0 / 6$ & $1 / 6$ & $1 / 6$ & $1 / 6$ & $0 / 6$ \\
\hline & 52 & $6 / 6 * *$ & $0 / 6$ & $0 / 6$ & $0 / 6$ & $1 / 6$ & $6 / 6$ & $4 / 6$ & $0 / 6$ \\
\hline & 78 & $4 / 8^{*}$ & $1 / 8$ & $2 / 8$ & $1 / 8$ & $8 / 8$ & $8 / 8$ & $6 / 8$ & $2 / 8$ \\
\hline & 104 & $15 / 30 * *$ & $1 / 27$ & $18 / 30$ & $8 / 27$ & $29 / 30$ & $26 / 27$ & $24 / 30$ & $0 / 27 * *$ \\
\hline & Total & $15 / 40 * *$ & $1 / 40$ & $21 / 40$ & $10 / 40$ & $35 / 40$ & $38 / 40$ & $24 / 40$ & $0 / 40 * *$ \\
\hline \multirow[t]{5}{*}{50} & 26 & $6 / 6^{* *}$ & $6 / 6^{* *}$ & $0 / 6$ & $0 / 6$ & $1 / 6$ & $3 / 6$ & $1 / 6$ & $0 / 6$ \\
\hline & 52 & $6 / 6^{* *}$ & $6 / 6^{* *}$ & $0 / 6$ & $0 / 6$ & $0 / 6$ & $6 / 6$ & $4 / 6$ & $0 / 6$ \\
\hline & 78 & $8 / 8 * *$ & $7 / 7 * *$ & $8 / 8 * *$ & $1 / 7$ & $8 / 8$ & $7 / 7$ & $8 / 8$ & $0 / 7$ \\
\hline & 104 & $33 / 36^{* *}$ & $31 / 32 * *$ & $30 / 36$ & $16 / 32 * *$ & $34 / 36$ & $30 / 32$ & $27 / 36$ & $8 / 32$ \\
\hline & Total & $35 / 40 * *$ & $34 / 40 * *$ & $34 / 40 * *$ & $19 / 40 * *$ & $38 / 40$ & $38 / 40$ & $27 / 40$ & $8 / 40$ \\
\hline \multirow[t]{5}{*}{500} & 26 & $6 / 6^{* *}$ & $6 / 6^{* *}$ & $4 / 6^{*}$ & $0 / 6$ & $0 / 6$ & $2 / 6$ & $0 / 6$ & $0 / 6$ \\
\hline & 52 & $6 / 6 * *$ & $6 / 6^{* *}$ & $6 / 6 * *$ & $2 / 6$ & $0 / 6$ & $0 / 6^{*}$ & $0 / 6 * *$ & $0 / 6$ \\
\hline & 78 & $7 / 7 * *$ & $8 / 8 * *$ & $7 / 7 * *$ & $8 / 8^{* *}$ & $3 / 7 *$ & $6 / 8$ & $0 / 7 * *$ & $0 / 8$ \\
\hline & 104 & $31 / 33 * *$ & $31 / 33 * *$ & $33 / 33 * *$ & $31 / 33 * *$ & $29 / 33$ & $23 / 33^{*}$ & $13 / 33 * *$ & $11 / 33$ \\
\hline & Total & $38 / 40 * *$ & $37 / 40 * *$ & $38 / 40 * *$ & $37 / 40 * *$ & $34 / 40$ & $27 / 40 * *$ & $14 / 40^{*}$ & $11 / 40$ \\
\hline
\end{tabular}

a: Total number of animals examined (No. of scheduled deaths after 104 weeks + No. of unscheduled deaths).

*, **: Significantly different from control at $\mathrm{p}<0.05$ and $\mathrm{p}<0.01$, respectively (Fisher's exact test).

Table 14. Number of Altered Hepatocellular Foci (AHF) in F344 Rats from a 2-Year Feeding Study of DDT

\begin{tabular}{|c|c|c|c|c|c|c|c|c|c|}
\hline \multirow{3}{*}{$\begin{array}{l}\text { Dose } \\
\text { (ppm) }\end{array}$} & \multirow{3}{*}{$\begin{array}{c}\text { Duration } \\
\text { (weeks) }\end{array}$} & \multirow{2}{*}{\multicolumn{2}{|c|}{$\begin{array}{l}\text { No. of rats } \\
\text { examined }\end{array}$}} & \multicolumn{6}{|c|}{ No. of AHF $\left(\right.$ No. $\left./ \mathrm{cm}^{2}\right):$ Mean \pm SD } \\
\hline & & & & \multicolumn{2}{|c|}{ Eosinophilic } & \multicolumn{2}{|c|}{ Tigroid basophilic } & \multicolumn{2}{|c|}{ Clear cell } \\
\hline & & Male & Female & Male & Female & Male & Female & Male & Female \\
\hline \multirow[t]{4}{*}{0} & 26 & 6 & 6 & $0.0 \pm 0.0$ & $0.0 \pm 0.0$ & $0.4 \pm 0.6$ & $0.6 \pm 0.9$ & $0.0 \pm 0.0$ & $0.0 \pm 0.0$ \\
\hline & 52 & 6 & 6 & $0.0 \pm 0.0$ & $0.0 \pm 0.0$ & $1.0 \pm 1.3$ & $1.6 \pm 1.6$ & $1.6 \pm 1.1$ & $0.0 \pm 0.0$ \\
\hline & 78 & 8 & 8 & $0.1 \pm 0.4$ & $0.0 \pm 0.0$ & $2.2 \pm 1.3$ & $9.3 \pm 3.7$ & $0.8 \pm 0.8$ & $0.5 \pm 0.7$ \\
\hline & 104 & 10 & 10 & $1.5 \pm 1.5$ & $0.6 \pm 1.0$ & $6.5 \pm 4.8$ & $11.0 \pm 5.3$ & $1.9 \pm 1.7$ & $0.8 \pm 0.9$ \\
\hline \multirow[t]{4}{*}{5} & 26 & 6 & 6 & $0.0 \pm 0.0$ & $0.0 \pm 0.0$ & $0.2 \pm 0.4$ & $0.3 \pm 0.7$ & $0.2 \pm 0.4$ & $0.0 \pm 0.0$ \\
\hline & 52 & 6 & 6 & $0.0 \pm 0.0$ & $0.0 \pm 0.0$ & $0.3 \pm 0.8$ & $4.7 \pm 1.7$ & $1.3 \pm 1.4$ & $0.0 \pm 0.0$ \\
\hline & 78 & 8 & 8 & $0.5 \pm 0.9$ & $0.2 \pm 0.5$ & $2.8 \pm 1.7$ & $10.0 \pm 5.3$ & $1.4 \pm 1.2$ & $0.6 \pm 1.1$ \\
\hline & 104 & 10 & 10 & $2.0 \pm 1.8$ & $0.6 \pm 0.7$ & $5.1 \pm 4.6$ & $14.2 \pm 4.0$ & $2.4 \pm 2.0$ & $0.0 \pm 0.0$ \\
\hline \multirow[t]{4}{*}{50} & 26 & 6 & 6 & $0.0 \pm 0.0$ & $0.0 \pm 0.0$ & $0.4 \pm 0.9$ & $0.8 \pm 0.8$ & $0.2 \pm 0.4$ & $0.0 \pm 0.0$ \\
\hline & 52 & 6 & 6 & $0.0 \pm 0.0$ & $0.0 \pm 0.0$ & $0.0 \pm 0.0$ & $5.5 \pm 1.6^{*}$ & $1.1 \pm 1.1$ & $0.0 \pm 0.0$ \\
\hline & 78 & 8 & 7 & $3.1 \pm 1.5^{*}$ & $0.6 \pm 1.5$ & $2.0 \pm 1.9$ & $8.7 \pm 4.1$ & $1.2 \pm 0.5$ & $0.0 \pm 0.0$ \\
\hline & 104 & 10 & 10 & $8.9 \pm 5.1^{* *}$ & $1.0 \pm 2.2$ & $3.8 \pm 2.3$ & $17.6 \pm 4.5$ & $2.9 \pm 1.2$ & $0.3 \pm 0.7$ \\
\hline \multirow[t]{4}{*}{500} & 26 & 6 & 6 & $0.8 \pm 0.7^{* *}$ & $0.0 \pm 0.0$ & $0.0 \pm 0.0$ & $0.5 \pm 0.8$ & $0.0 \pm 0.0$ & $0.0 \pm 0.0$ \\
\hline & 52 & 6 & 6 & $3.7 \pm 1.6^{* *}$ & $1.4 \pm 2.1$ & $0.0 \pm 0.0$ & $0.0 \pm 0.0$ & $0.0 \pm 0.0^{*}$ & $0.0 \pm 0.0$ \\
\hline & 78 & 7 & 8 & $9.2 \pm 3.1 * *$ & $12.1 \pm 3.5^{* *}$ & $0.5 \pm 0.6$ & $1.0 \pm 0.7 * *$ & $0.0 \pm 0.0$ & $0.0 \pm 0.0$ \\
\hline & 104 & 10 & 10 & $10.4 \pm 3.1^{* *}$ & $9.8 \pm 3.8^{* *}$ & $2.1 \pm 1.0$ & $1.3 \pm 1.3^{*}$ & $0.4 \pm 0.6^{*}$ & $1.0 \pm 1.3$ \\
\hline
\end{tabular}

*, **: Significantly different from control at $\mathrm{p}<0.05$ and $\mathrm{p}<0.01$, respectively (Fisher's exact test).

a carrier of genetic messages ${ }^{27}$. In our 2-year study, the incidence of hepatocellular tumors, especially carcinomas, was much higher in males than females. This sex difference might be partly due to the higher concentrations of DDT and its metabolites (DDE and DDD) in the liver of males ${ }^{11}$ which may result in higher production of oxidative stress through metabolic activation. In fact, the levels of LPO and 8-OHdG were much higher in males than females. In addition, the higher incidence of spontaneous eosinophilic foci in male F344 rats $^{28}$ may contribute to the sex difference. In other words, the male liver may have a preferable microenvironment for occurrence of eosinophilic AHF. The 
Table 15. Mean Area of Altered Hepatocellular Foci (AHF) in F344 Rats from a 2-Year Feeding Study of DDT

\begin{tabular}{|c|c|c|c|c|c|c|c|c|c|}
\hline \multirow{3}{*}{$\begin{array}{l}\text { Dose } \\
(\mathrm{ppm})\end{array}$} & \multirow{3}{*}{$\begin{array}{l}\text { Duration } \\
\text { (weeks) }\end{array}$} & \multirow{2}{*}{\multicolumn{2}{|c|}{$\begin{array}{c}\text { No. of rats } \\
\text { examined }\end{array}$}} & \multicolumn{6}{|c|}{ Mean area of AHF $\left(\mathrm{mm}^{2}\right)$ : Mean \pm SD (No. of rats with AHF) } \\
\hline & & & & \multicolumn{2}{|c|}{ Eosinophilic } & \multicolumn{2}{|c|}{ Tigroid basophilic } & \multicolumn{2}{|c|}{ Clear cell } \\
\hline & & Male & Female & Male & Female & Male & Female & Male & Female \\
\hline \multirow[t]{4}{*}{0} & 26 & 6 & 6 & - & - & $0.02 \pm 0.004(2)$ & $0.03 \pm 0.004(2)$ & - & - \\
\hline & 52 & 6 & 6 & - & - & $0.06 \pm 0.05(3)$ & $0.13 \pm 0.09(4)$ & $0.02 \pm 0.02(5)$ & - \\
\hline & 78 & 8 & 8 & $0.16(1)$ & - & $0.09 \pm 0.03(8)$ & $0.11 \pm 0.03(8)$ & $0.05 \pm 0.02(5)$ & $0.04 \pm 0.01(3)$ \\
\hline & 104 & 10 & 10 & $0.33 \pm 0.16(8)$ & $0.46 \pm 0.18(3)$ & $0.16 \pm 0.04(9)$ & $0.17 \pm 0.08(10)$ & $0.08 \pm 0.04(9)$ & $0.09 \pm 0.08(5)$ \\
\hline \multirow[t]{4}{*}{5} & 26 & 6 & 6 & - & - & $0.03(1)$ & $0.02(1)$ & $0.03(1)$ & - \\
\hline & 52 & 6 & 6 & - & - & $0.03(1)$ & $0.09 \pm 0.03(6)$ & $0.03 \pm 0.01(4)$ & - \\
\hline & 78 & 8 & 8 & $0.60 \pm 0.63(2)$ & $0.04(1)$ & $0.09 \pm 0.05(8)$ & $0.08 \pm 0.03(8)^{*}$ & $0.05 \pm 0.03(6)$ & $0.05 \pm 0.02(2)$ \\
\hline & 104 & 10 & 10 & $0.44 \pm 0.57(7)$ & $0.38 \pm 0.39(4)$ & $0.11 \pm 0.04(9)^{* *}$ & $0.14 \pm 0.03(10)$ & $0.08 \pm 0.04(10)$ & - \\
\hline \multirow[t]{4}{*}{50} & 26 & 6 & 6 & - & - & $0.06(1)$ & $0.03 \pm 0.003(3)$ & $0.03(1)$ & - \\
\hline & 52 & 6 & 6 & - & - & - & $0.09 \pm 0.02(6)$ & $0.05 \pm 0.02(4)$ & - \\
\hline & 78 & 8 & 7 & $0.21 \pm 0.17(8)$ & $0.32(1)$ & $0.25 \pm 0.49(8)$ & $0.10 \pm 0.04(7)$ & $0.09 \pm 0.06(8)$ & - \\
\hline & 104 & 10 & 10 & $0.58 \pm 0.54(9)$ & $0.35 \pm 0.21(2)$ & $0.11 \pm 0.06(9)^{*}$ & $0.15 \pm 0.03(10)$ & $0.18 \pm 0.24(10)$ & $0.05 \pm 0.03(2)$ \\
\hline \multirow[t]{4}{*}{500} & 26 & 6 & 6 & $0.05 \pm 0.03(4)$ & - & - & $0.07 \pm 0.06(2)$ & - & - \\
\hline & 52 & 6 & 6 & $0.06 \pm 0.03(6)$ & $0.06 \pm 0.02(2)$ & - & - & - & - \\
\hline & 78 & 7 & 8 & $0.32 \pm 0.18(7)$ & $0.26 \pm 0.13(8)$ & $0.07 \pm 0.02(3)$ & $0.09 \pm 0.06(6)$ & - & - \\
\hline & 104 & 10 & 10 & $0.90 \pm 0.43(10)^{* *}$ & $1.02 \pm 0.70(10)$ & $0.17 \pm 0.10(10)$ & $0.08 \pm 0.02(8) * *$ & $0.41 \pm 0.53(4)$ & $0.03 \pm 0.01(4)$ \\
\hline
\end{tabular}

-: Not available because of no AHF.

*, **: Significantly different from control at $\mathrm{p}<0.05$ and $\mathrm{p}<0.01$, respectively (Fisher's exact test).

Table 16. Area Fraction of Liver Occupied by Altered Hepatocellular Foci (AHF) in F344 Rats from a 2-Year Feeding Study of DDT

\begin{tabular}{|c|c|c|c|c|c|c|c|c|c|}
\hline \multirow{3}{*}{$\begin{array}{l}\text { Dose } \\
(\mathrm{ppm})\end{array}$} & \multirow{3}{*}{$\begin{array}{l}\text { Duration } \\
\text { (weeks) }\end{array}$} & \multirow{2}{*}{\multicolumn{2}{|c|}{$\begin{array}{l}\text { No. of rats } \\
\text { examined }\end{array}$}} & \multicolumn{6}{|c|}{ Area fraction occupied by AHF (\%): Mean \pm SD } \\
\hline & & & & \multicolumn{2}{|c|}{ Eosinophilic } & \multicolumn{2}{|c|}{ Tigroid basophilic } & \multicolumn{2}{|c|}{ Clear cell } \\
\hline & & Male & Female & Male & Female & Male & Female & Male & Female \\
\hline \multirow[t]{4}{*}{0} & 26 & 6 & 6 & $0.00 \pm 0.00$ & $0.00 \pm 0.00$ & $0.01 \pm 0.01$ & $0.02 \pm 0.03$ & $0.00 \pm 0.00$ & $0.00 \pm 0.00$ \\
\hline & 52 & 6 & 6 & $0.00 \pm 0.00$ & $0.00 \pm 0.00$ & $0.08 \pm 0.16$ & $0.26 \pm 0.42$ & $0.04 \pm 0.04$ & $0.00 \pm 0.00$ \\
\hline & 78 & 8 & 8 & $0.02 \pm 0.05$ & $0.00 \pm 0.00$ & $0.18 \pm 0.10$ & $1.06 \pm 0.54$ & $0.04 \pm 0.05$ & $0.02 \pm 0.03$ \\
\hline & 104 & 10 & 10 & $0.51 \pm 0.41$ & $0.25 \pm 0.44$ & $0.98 \pm 0.70$ & $1.82 \pm 1.16$ & $0.16 \pm 0.18$ & $0.08 \pm 0.13$ \\
\hline \multirow[t]{4}{*}{5} & 26 & 6 & 6 & $0.00 \pm 0.00$ & $0.00 \pm 0.00$ & $0.005 \pm 0.012$ & $0.01 \pm 0.01$ & $0.01 \pm 0.01$ & $0.00 \pm 0.00$ \\
\hline & 52 & 6 & 6 & $0.00 \pm 0.00$ & $0.00 \pm 0.00$ & $0.01 \pm 0.02$ & $0.41 \pm 0.26$ & $0.04 \pm 0.04$ & $0.00 \pm 0.00$ \\
\hline & 78 & 8 & 8 & $0.31 \pm 0.77$ & $0.01 \pm 0.02$ & $0.21 \pm 0.11$ & $0.85 \pm 0.52$ & $0.06 \pm 0.07$ & $0.02 \pm 0.04$ \\
\hline & 104 & 10 & 10 & $1.07 \pm 1.85$ & $0.20 \pm 0.38$ & $0.54 \pm 0.42$ & $1.93 \pm 0.45$ & $0.19 \pm 0.16$ & $0.00 \pm 0.00^{*}$ \\
\hline \multirow[t]{4}{*}{50} & 26 & 6 & 6 & $0.00 \pm 0.00$ & $0.00 \pm 0.00$ & $0.02 \pm 0.05$ & $0.03 \pm 0.03$ & $0.005 \pm 0.011$ & $0.00 \pm 0.00$ \\
\hline & 52 & 6 & 6 & $0.00 \pm 0.00$ & $0.00 \pm 0.00$ & $0.00 \pm 0.00$ & $0.47 \pm 0.12$ & $0.06 \pm 0.09$ & $0.00 \pm 0.00$ \\
\hline & 78 & 8 & 7 & $0.60 \pm 0.39 *$ & $0.18 \pm 0.48$ & $0.34 \pm 0.48$ & $0.89 \pm 0.53$ & $0.12 \pm 0.08$ & $0.00 \pm 0.00$ \\
\hline & 104 & 10 & 10 & $5.10 \pm 4.74 *$ & $0.32 \pm 0.71$ & $0.41 \pm 0.32$ & $2.54 \pm 0.76$ & $0.42 \pm 0.52$ & $0.02 \pm 0.04$ \\
\hline \multirow[t]{4}{*}{500} & 26 & 6 & 6 & $0.04 \pm 0.05 * *$ & $0.00 \pm 0.00$ & $0.00 \pm 0.00$ & $0.03 \pm 0.07$ & $0.00 \pm 0.00$ & $0.00 \pm 0.00$ \\
\hline & 52 & 6 & 6 & $0.23 \pm 0.09 * *$ & $0.08 \pm 0.14$ & $0.00 \pm 0.00$ & $0.00 \pm 0.00$ & $0.00 \pm 0.00$ & $0.00 \pm 0.00$ \\
\hline & 78 & 7 & 8 & $2.72 \pm 1.05 * *$ & $3.01 \pm 1.84 * *$ & $0.03 \pm 0.05^{*}$ & $0.10 \pm 0.10 * *$ & $0.00 \pm 0.00$ & $0.00 \pm 0.00$ \\
\hline & 104 & 10 & 10 & $9.25 \pm 6.28 * *$ & $8.25 \pm 4.53 * *$ & $0.31 \pm 0.19$ & $0.10 \pm 0.09 * *$ & $0.14 \pm 0.30$ & $0.03 \pm 0.03$ \\
\hline
\end{tabular}

*,**: Significantly different from control at $\mathrm{p}<0.05$ and $\mathrm{p}<0.01$, respectively (Fisher's exact test).

reduced body weight gain in the high-dose group which was more evident in females than males ${ }^{11}$ was also considered as an influential factor for tumor development, but it seems to have no correlation with the occurrence of hepatocellular tumors in $\mathrm{F} 344$ rats $^{29}$.

\section{Gene Expression in Target Sites}

Analysis of gene expression by microarray following laser-captured microdissection was performed on the target tissues including hepatocellular hypertrophy, eosinophilic $\mathrm{AHF}$, and hepatocellular adenoma observed in the high-dose and/or control rats. The results are summarized in Table 18.

As shown in Table 18, various genes relating to cell proliferation, apoptosis or anti-oxidative function were upregulated or downregulated in the target sites and the most prominent changes were noted in the eosinophilic AHF from the high-dose rat. These gene expressions might be corresponding changes to the mitogenic activity of DDT and/or oxidative stress generated though the metabolic 
Table 17. Incidence of Hepatocellular Tumors in F344 Rats from a 2-Year Feeding Study of DDT

\begin{tabular}{|c|c|c|c|c|c|}
\hline \multirow{2}{*}{$\begin{array}{l}\text { Dose } \\
\text { (ppm) }\end{array}$} & \multirow{2}{*}{ Duration (weeks) } & \multicolumn{2}{|c|}{ Adenoma } & \multicolumn{2}{|c|}{ Carcinoma } \\
\hline & & Male & Female & Male & Female \\
\hline \multirow[t]{5}{*}{0} & 26 & $0 / 6$ & $0 / 6$ & $0 / 6$ & $0 / 6$ \\
\hline & 52 & $0 / 6$ & $0 / 6$ & $0 / 6$ & $0 / 6$ \\
\hline & 78 & $0 / 8$ & $0 / 8$ & $0 / 8$ & $0 / 8$ \\
\hline & 104 & $0 / 35$ & $0 / 33$ & $0 / 35$ & $0 / 33$ \\
\hline & Total $^{\mathrm{a}}$ & $0 / 40$ & $0 / 40$ & $0 / 40$ & $0 / 40$ \\
\hline \multirow[t]{5}{*}{5} & 26 & $0 / 6$ & $0 / 6$ & $0 / 6$ & $0 / 6$ \\
\hline & 52 & $0 / 6$ & $0 / 6$ & $0 / 6$ & $0 / 6$ \\
\hline & 78 & $0 / 8$ & $0 / 8$ & $0 / 8$ & $0 / 8$ \\
\hline & 104 & $0 / 30$ & $0 / 27$ & $0 / 30$ & $0 / 27$ \\
\hline & Total & $0 / 40$ & $0 / 40$ & $0 / 40$ & $0 / 40$ \\
\hline \multirow[t]{5}{*}{50} & 26 & $0 / 6$ & $0 / 6$ & $0 / 6$ & $0 / 6$ \\
\hline & 52 & $0 / 6$ & $0 / 6$ & $0 / 6$ & $0 / 6$ \\
\hline & 78 & $0 / 8$ & $0 / 7$ & $0 / 8$ & $0 / 7$ \\
\hline & 104 & $5 / 36^{*}$ & $0 / 32$ & $0 / 36$ & $0 / 32$ \\
\hline & Total & $5 / 40 *$ & $0 / 40$ & $0 / 40$ & $0 / 40$ \\
\hline \multirow[t]{5}{*}{500} & 26 & $0 / 6$ & $0 / 6$ & $0 / 6$ & $0 / 6$ \\
\hline & 52 & $0 / 6$ & $0 / 6$ & $0 / 6$ & $0 / 6$ \\
\hline & 78 & $6 / 7 * *$ & $1 / 8$ & $0 / 7$ & $0 / 8$ \\
\hline & 104 & $20 / 33 * *$ & $16 / 33 * *$ & $13 / 33 * *$ & $2 / 33$ \\
\hline & Total & $22 / 40 * *$ & $16 / 40 * *$ & $14 / 40 * *$ & $2 / 40$ \\
\hline
\end{tabular}

Table 18. Microarray Assay of Hepatoproliferative Lesions in Male F344 Rats from a 2-Year Feeding Study of DDT

\begin{tabular}{|c|c|c|c|c|c|}
\hline \multirow{3}{*}{ Gene / protein name } & \multirow{3}{*}{ Function } & \multicolumn{4}{|c|}{ Fold change (ratio to normal control value)* } \\
\hline & & \multirow{2}{*}{$\frac{\text { Hypertorophy }}{500 \mathrm{ppm}}$} & \multicolumn{2}{|c|}{ Eosinophilic AHF } & \multirow{2}{*}{$\begin{array}{l}\text { Adenoma } \\
500 \mathrm{ppm}\end{array}$} \\
\hline & & & $0 \mathrm{ppm}$ & $500 \mathrm{ppm}$ & \\
\hline Protein kinase C-eta & Cell proliferation (CP) & 3.6 & 3.2 & 3.1 & 2.5 \\
\hline Uncouplingprotein 2, mitochondrial & $\mathrm{CP}$ & 2.0 & - & 2.0 & - \\
\hline Phosphorylase B kinase catalytic subunit & $\mathrm{CP}$ & - & - & 2.2 & - \\
\hline Guanine nucleotide-binding protein G-s, $\alpha$ subunit & $\mathrm{CP}$ & 2.5 & - & 2.6 & 2.3 \\
\hline G protein $\gamma-5$ subunit & $\mathrm{CP}$ & 2.0 & - & 3.5 & - \\
\hline CDK106 & CP (Downreg. TGF- $\beta$ ) & - & - & 2.0 & 2.0 \\
\hline Follistatin & CP (Downreg. TGF- $\beta$ ) & - & - & 0.5 & - \\
\hline Lipoprotein lipase & $\mathrm{CP}(\mathrm{TGF}-\beta)$ & - & - & 0.5 & - \\
\hline Ubiquitin-conjugating enzyme E2D3 & CP (Cell cycle) & - & - & 0.5 & - \\
\hline p53-activated gene 608 & Apoptosis (Apo) & - & - & 2.3 & 2.0 \\
\hline Sodium channel $\beta 2$ & Apo (suppre) & 2.9 & 2.2 & 3.2 & 2.4 \\
\hline Protein disulfide isomerase A6 precursor & Apo (suppre) & - & - & 0.5 & - \\
\hline Protein phosphatase type $1 \alpha$, catalytic subunit & Apo (suppre) & 0.5 & - & 0.4 & - \\
\hline Potassium channel gene 1 (Kir6) & Anti-oxidative & 3.2 & 2.7 & 3.1 & 2.5 \\
\hline Dopamine receptor D2 & Anti-oxidative & 2.7 & 3.0 & 2.1 & 2.2 \\
\hline
\end{tabular}

*: Values of 2.0 or more and 0.5 or less represent upregulation and downregulation, respectively.

-: Within normal range

activation. However, we need further accumulation of gene expression data to arrive at conclusion since the information from our results is quite limited.

\section{Conclusion}

Our study results indicate that DDT may induce eosinophilic AHF as a result of oxidative DNA damage of hepatocytes and promote the progression of the preneoplastic lesions into hepatocellular tumors in 
combination with its mitogenic activity and inhibitory effect on GJIC. The production of oxidative stress (increases in LPO and 8-OHdG) which might be secondary to metabolic activation could be a key factor in hepatocarcinogenesis by DDT and may play an important role in tumor promotion and progression (malignant transformation).

Acknowledgments: These studies were supported by a grant from the Ministry of Agriculture, Forestry and Fisheries. We are grateful to our colleagues in the Toxicology and Chemictry Divisions of the Institute of Environmental Toxicology for their valuable suggestions, cooperation and/or technical support in this work.

\section{References}

1. International Agency for Research on Cancer (IARC). Occupational Exposures in Insecticide Application, and Some Pesticides. IARC Monographs on the Evaluation of Carcinogenic Risks to Humans, Vol. 53. Lyon. 179-249. 1991.

2. Flodström S, Hemming H, Wärngård L, and Ahlborg UG. Promotion of altered hepatic foci development in rat liver, cytochrome P450 enzyme induction and inhibition of cellcell communication by DDT and some structurally related organohalogen pesticides. Carcinogenesis. 11: 1413-1417. 1990.

3. Lubet RA, Dragnev KH, Chauhan DP, Nims RW, Diwan BA, Ward JM, Jones CR, Rice JM, and Miller MS. A pleiotropic response to phenobarbital-type enzyme inducers in the F344/NCr rat. Biochem Pharmacol. 43: 1067-1078. 1992.

4. Nims RW, Lubet RA, Fox SD, Jones CR, Thomas PE, Reddy AB, and Kocarek TA. Comparative pharmacodynamics of CYP2B induction by DDT, DDE, and DDD in male rat liver and cultured rat hepatocytes. J Toxicol Environ Health. 53: 455-457. 1998.

5. Sierra-Santoyo A, Hernández M, Albores A, and Cebrián ME. Sex-dependent regulation of hepatic cytochrome P-450 by DDT. Toxicol Sci. 54: 81-87. 2000.

6. Jansen LAM and Jongen WMF. The use of initiated cells as a test system for detection of inhibitors of gap junctional intercellular communication. Carcinogenesis. 17: 333-339. 1996.

7. Klaunig JE and Ruch RJ. Strain and species effects on the inhibition of hepatocyte intercellular communication by liver tumor promoters. Cancer Lett. 36: 161-168. 1987.

8. Krutovskikh VA, Mesnil M, Mazzoleni G, and Yamasaki H. Inhibition of rat liver gap junction intercellular communication by tumor-promoting agents in vivo. Lab Invest. 72: 571-577. 1995.

9. Williams GM. Epigenetic mechanisms of liver tumor promotion. In: Mouse Liver Carcinogenesis: Mechanisms and Species Comparisons, DE Stevenson, RM McClain, JA Popp, TJ Slaga, JM Ward, and HC Pitot (eds). Wiley-Liss, New York. 131-145. 1990.

10. Trosko JE and Ruch RJ. Gap junctions as targets for cancer chemoprevention and chemotherapy. Curr Drug Targets. 3: 465-482. 2002.

11. Harada T, Yamaguchi S, Ohtsuka R, Takeda M, Fujisawa H,
Yoshida T, Enomoto A, Chiba Y, Fukumori J, Kojima S, Tomiyama N, Saka M, Ozaki M, and Maita K. Mechanisms of promotion and progression of preneoplastic lesions in hepatocarcinogenesis by DDT in F344 rats. Toxicol Pathol. 31: 87-98. 2003.

12. Parke DV and Ioannides C. Role of cytochromes P-450 in mouse liver tumor production. In: Mouse Liver Carcinogenesis: Mechanisms and Species Comparisons, DE Stevenson, RM McClain, JA Popp, TJ Slaga, JM Ward, and HC Pitot (eds). Wiley-Liss, New York. 215-230. 1990.

13. Parkinson A. An overview of current cytochrome P450 technology for assessing the safety and efficacy of new materials. Toxicol Pathol. 24: 45-57. 1996.

14. Simic MG. Mechanisms of inhibition of free-radical processes in mutagenesis and carcinogenesis. Mutation Res. 202: 377-386. 1988.

15. Clayson DB, Mehta R, and Iverson F. Oxidative DNA damage - The effects of certain genotoxic and operationally non-genotoxic carcinogens. Mutation Res. 317: 25-42. 1994.

16. Butterworth BE, Conolly RB, and Morgan KT. A strategy for establishing mode of action of chemical carcinogens as a guide for approaches to risk assessments. Cancer Lett. 93: 129-146. 1995.

17. Schulte-Hermann R, Schuppler J, Timmermann-Trosiener I, Ohde G, Bursch W, and Berger $\mathrm{H}$. The role of growth of normal and preneoplastic cell populations for tumor promotion in rat liver. Environ Health Perspect. 50: 185194. 1983.

18. Schulte-Hermann R, Bursch W, Grasl-Kranpp B, Huber W, and Parzefall W. Nongenotoxic carcinogenesis in the liver. In: Nongenotoxic Carcinogenesis, A Cockburn and L Smith L (eds). Springer-Verlag, Berlin. 109-120. 1994.

19. Büsser M-T and Lutz WK. Stimulation of DNA synthesis in rat and mouse liver by various tumor promoters. Carcinogenesis. 8: 1433-1437. 1987.

20. Beer DG and Neveu MJ. Proto-oncogene and gap-junction protein expression in rodent liver neoplasms. In: Mouse Liver Carcinogenesis: Mechanisms and Species Comparisons, DE Stevenson, RM McClain, JA Popp, TJ Slaga, JM Ward, HC Pitot (eds). Wiley-Liss, New York. 293-309. 1990.

21. Whysner J, Ross PM, and Williams GM. Phenobarbital mechanistic data and risk assessment: enzyme induction, enhanced cell proliferation, and tumor promotion. Phamacol Ther. 71: 153-191. 1996.

22. Yamasaki H. Role of disrupted gap junctional intercellular communication in detection and characterization of carcinogens. Mutation Res. 365: 91-105. 1996.

23. Loewenstein WR. Junctional intercellular communication and the control of growth. Biochim Biophys Acta. 560: 165. 1979.

24. Ströbel P, Klimek F, Zerban H, Kopp-Schneider A, and Bannasch P. Xenomorphic hepatocellular precursors and neoplastic progression of tigroid cell foci induced in rats with low doses of N-nitrosomorpholine. Carcinogenesis. 19: 2069-2080. 1998.

25. Bannasch P, Zerban H, and Hacker HJ. Foci of altered hepatocytes, rat. In: Monographs on Pathology of Laboratory Animals, Digestive System, TC Jones, U Mohr, and RD Hunt (eds). Springer-Verlag, Berlin. 10-30. 1985.

26. Harada T, Maronpot RR, Morris RW, and Boorman GA. 
Observations on altered hepatocellular foci in National Toxicology Program 2-year carcinogenicity studies in rats. Toxicol Pathol. 17: 690-708. 1989.

27. Toh YC. Physiological and biochemical reviews of sex differences and carcinogenesis with particular reference to the liver. Adv Cancer Res. 18: 155-209. 1973.

28. Harada T, Maronpot RR, Morris RW, Stitzel KA, and
Boorman GA. Morphological and stereological characterization of hepatic foci of cellular alteration in control Fischer 344 rats. Toxicol Pathol. 17: 579-593. 1989.

29. Thurman JD, Bucci TJ, Hart RW, and Turturro A. Survival, body weight, and spontaneous neoplasms in ad libitum-fed and food-restricted Fischer-344 rats. Toxicol Pathol. 22: 19. 1994. 